Canadian

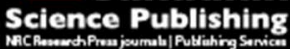

Applied Physiology, Nutrition, and Metabolism Physiologie appliquée, nutrition et métabolisme

\title{
Waist circumference is associated with liver fat in black and white adolescents.
}

\begin{tabular}{|r|l|}
\hline Journal: & Applied Physiology, Nutrition, and Metabolism \\
\hline Manuscript ID & apnm-2016-0410.R1 \\
\hline Manuscript Type: & Article \\
\hline Complete List of Authors: & $\begin{array}{l}\text { Lee, SoJung; Childrens Hospital of Pittsburgh, University of Pittsburgh } \\
\text { School of Medicine; Kyung Hee University - Global Campus, Sports } \\
\text { Medicine, Graduate School of Physical Education } \\
\text { Kuk, Jennifer; York University, } \\
\text { Boesch, Chris; University of Bern, 4. Department of Clinical Research, } \\
\text { Magnetic Resonance Spectroscopy, and Methodology } \\
\text { Arslanian, Silva; University of Pittsburgh School of Medicine, Children's } \\
\text { Hospital of Pittsburgh }\end{array}$ \\
\hline Keyword: & $\begin{array}{l}\text { childhood obesity, race, waist circumference, visceral fat < waist } \\
\text { circumference, liver fat }\end{array}$ \\
\hline \multicolumn{2}{|l}{} \\
\hline
\end{tabular}




\section{Waist circumference is associated with liver fat in black and white adolescents}

SoJung Lee, $\mathrm{PhD}^{1,2}$, Jennifer L. Kuk, $\mathrm{PhD}^{4}$, Chris Boesch, MD, $\mathrm{PhD}^{5}$, Silva Arslanian, MD ${ }^{1,3}$

1. Division of Weight Management \& Wellness, Children's Hospital of Pittsburgh of UPMC, University of Pittsburgh School of Medicine, Pittsburgh, Pennsylvania, USA

2. Department of Sports Medicine, Graduate School of Physical Education, Kyung Hee University, Yongin, Korea

3. Division of Pediatric Endocrinology, Metabolism and Diabetes Mellitus, Children's Hospital of Pittsburgh of UPMC, University of Pittsburgh School of Medicine, Pittsburgh, Pennsylvania, USA

4. School of Kinesiology and Health Science, York University, Toronto, ON, Canada

5. Department of Clinical Research, Magnetic Resonance Spectroscopy, and Methodology, University of Bern, Bern, CH-3010, Switzerland

Running Title: Waist circumference and liver fat in obese adolescents

Word count: 2,655

\section{Address correspondence to:}

SoJung Lee, $\mathrm{PhD}$

Division of Weight Management \& Wellness

Children's Hospital of Pittsburgh of UPMC

University of Pittsburgh School of Medicine

Faculty Pavilion Sixth Floor (6104)

40045 th Street, Pittsburgh, PA 15224

Phone: (412) 692-5147

Fax: (412) 692-8531

E-mail: SoJung.Lee@chp.edu 


\section{ABSTRACT}

We examined whether waist circumference (WC) is associated with liver fat in black and white adolescents. Liver fat was measured using a $3 \mathrm{~T}$ proton magnetic resonance spectroscopy $\left({ }^{1} \mathrm{H}-\right.$ MRS) in 152 overweight/obese adolescents (94 black and 58 white, $\mathrm{BMI} \geq 85^{\text {th }}$ percentile, $12-18$ yrs) without liver diseases or diabetes. WC was measured at the last rib. Total and visceral adipose tissue (VAT) were measured by DXA and MRI, respectively. The proportion of fatty liver (defined as liver fat $\geq 5.0 \%$ by $\left.{ }^{1} \mathrm{H}-\mathrm{MRS}\right)$ was lower $(P<0.01)$ in black adolescents $(5.3 \%)$ compared with their white peers (24.1\%). Despite similar age, BMI, WC and total adiposity (\%), blacks had lower $(P<0.01)$ VAT (59.0 versus $\left.81.3 \mathrm{~cm}^{2}\right)$, liver fat (1.6 versus $\left.3.5 \%\right)$ and ALT (17.2 versus $22.0 \mathrm{IU} / \mathrm{L}$ ) compared with their white peers. Independent of race, WC was associated with liver fat (black $r=0.43$, white $r=0.64$ ) in a similar magnitude to the association between VAT and liver fat (black $r=0.44$, white $r=0.51$ ) and these findings remained significant after controlling for age, gender, Tanner stage and total adiposity. In blacks, WC and gender (male) were independent $(P<0.01)$ predictors of liver fat, explaining $17.1 \%$ and $5.6 \%$ of the variance, respectively, while in whites WC was the single best predictor, explaining $40.8 \%$ of the variance in liver fat. These findings suggest that enlarged $\mathrm{WC}$ is a marker of increased liver fat in overweight/obese white and black adolescents.

\section{Word count: 236}

Key words: Childhood obesity, race, waist circumference, visceral fat, non-alcoholic fatty liver disease, proton magnetic resonance spectroscopy. 


\section{INTRODUCTION}

Non-alcoholic fatty liver disease (NAFLD) is common among obese children and adolescents (Lavine and Schwimmer 2004) and is strongly linked to insulin resistance, hypertension and dyslipidemia (Perseghin et al. 2006; Schwimmer et al. 2008; Kim et al. 2012; Wicklow et al. 2012; Brown et al. 2015). NAFLD ranges from simple steatosis (triglyceride accumulation within hepatocytes) to more advanced form of nonalcoholic steatohepatitis (NASH), which includes inflammation and liver cell injury (Lavine and Schwimmer 2004). A population-based liver histology study reports that the prevalence of NAFLD in the United States is substantially higher in obese adolescents (38\%) compared with their normal-weight peers (5\%) (Schwimmer et al. 2006). Racial disparity also exists, such that NAFLD is more prevalent in children and adolescent of Hispanic (11.8\%) and white $(8.6 \%)$ descent compared with their black peers (1.5\%) (Schwimmer et al. 2006).

Although liver biopsy remains the gold standard for evaluating NAFLD (Loomba et al. 2009), it is prone to selection bias due to small size of the liver biopsy specimens (Szczepaniak et al. 2005). Further, invasiveness and complications (e.g. abdominal pain, internal bleeding and infection) associated with the procedure may limit its use in the pediatric population, particularly for repeated measurements over time. Although imaging techniques such as computed tomography and ultrasound are routinely available in clinical practices, these methods rely on nonspecific attenuation of X-ray and doppler signals and, therefore lack sensitivity and accuracy in quantifying liver fat content, in particular detecting small amounts of liver fat in the case of ultrasound (Limanond et al. 2004; Mishra and Younossi 2007). Conversely, proton magnetic resonance spectroscopy $\left({ }^{1} \mathrm{H}-\mathrm{MRS}\right)$ does not use $\mathrm{x}$-rays or radiation and provides a quantitative measurement of liver fat by directly measuring the protons in the fatty acids of the triglycerides 
(Szczepaniak et al. 2005). To date, ${ }^{1} \mathrm{H}-\mathrm{MRS}$ is considered the optimal noninvasive imaging technique to quantify liver fat due to its high correlation with liver biopsy results and its sensitivity to detecting small amounts of liver fat (Longo et al. 1995). However, it is expensive and time consuming, and requires technical expertise to perform and to process the data, and thus is not routinely available in clinical settings.

Previously, we and others have shown that abdominal obesity evaluated by waist circumference (WC) is significantly associated with dysmetabolic syndrome and obesity-related comorbidities such as insulin resistance, high blood pressure (BP), unfavorable lipid profile, and atherogenic lipoprotein particle size and concentrations in children and adolescents (Janssen et al. 2005; Lee et al. 2006a; Lee et al. 2006b; Burns and Arslanian 2009). Further, we have shown that WC is an independent correlate of visceral adipose tissue (VAT) $(r=0.83)$ in youth $(8-17$ years) (Lee et al. 2006b). Given the strong associations between VAT and liver fat in adolescents (Lee et al. 2012), we examined whether WC is independently associated with increased liver fat in adolescents with obesity. To accomplish this, we employed a $3 \mathrm{~T}{ }^{1} \mathrm{H}-\mathrm{MRS}$ to quantify liver fat in a large sample of 152 overweight and obese black and white adolescents. 


\section{MATERIALS AND METHODS}

\section{Participants}

The study sample consisted of 94 black and 58 white overweight and obese adolescents $\left(\mathrm{BMI} \geq 85^{\text {th }}, 12-18\right.$ years) who were recruited between December 2008-April 2016 through advertisements in public transportation, posters placed on campus, and the Weight Management and Wellness Center at Children's Hospital of Pittsburgh (CHP) of UPMC. All subjects were non-smokers, non-diabetic, physically inactive and not taking medications known to affect study outcomes. None of the participants had liver diseases and consumed alcohol. Exclusion criteria included recent significant weight change (BMI $>2-3 \mathrm{~kg} / \mathrm{m}^{2}$ past 3 month prior to participation) or diagnosed endocrine disorders (e.g., polycystic ovary syndrome, type 2 diabetes) and syndromic obesity. Parental informed consent and child assent were obtained from all participants before participation.

Participants underwent a complete physical examination and medical history by a certified nurse practitioner at the Pediatric Clinical and Translational Research Center (PCTRC) at CHP. Participants self-identified as black or white and pubertal development was assessed according to Tanner criteria (genital development and pubic hair) (Tanner 1981). The study was approved by the University of Pittsburgh Institutional Review Board.

\section{Anthropometric measurement, and total and abdominal adipose tissue}

Anthropometric measurements were taken by trained research staff at the PCTRC. Body weight was measured to the nearest $0.1 \mathrm{~kg}$ and height was measured to the nearest $0.1 \mathrm{~cm}$. For WC measurement, participants were asked to expose the abdominal area and stand in an upright position with their feet side-by-side (shoulder width apart) and their arms across their chest. WC 
was measured immediately below the last rib at the end of expiration and the average of two measures was used in the analyses. Total body fat was assessed using lunar iDXA (GE Healthcare, Madison, WI, USA), and abdominal subcutaneous adipose tissue (ASAT) and visceral AT (VAT) were quantified at L4L5 using a magnetic resonance imaging. VAT was determined by delineating the intraabdominal cavity at the innermost aspect of the abdominal and oblique wall musculature and the anterior aspect of the vertebrae body as shown previously (Kuk et al. 2006). We previously reported that the intra-observer variability was $3.9 \%$ for visceral adipose tissue (AT) and 2.1\% for abdominal subcutaneous AT (Lee et al. 2010).

\section{Liver fat content by ${ }^{1} H-M R S$}

${ }^{1}$ H-MRS was performed with a 3.0 Tesla MR system (Siemens, Tim Trio, Erlangen, Germany) using a body matrix coil and a spine matrix (Siemens, Erlangen, Germany) using our established protocol described elsewhere (Lee et al. 2012; Lee et al. 2013). Fatty liver was defined as liver fat $[($ methylene lipid peak/ methylene lipid peak + water peak) $\mathrm{X} 100] \geq 5.0 \%$ as shown previously (Perseghin et al. 2006).

\section{Liver enzymes}

For liver enzymes, fasting blood samples were obtained after an overnight fast (minimum 8 hours). Samples were aliquoted and sent immediately to the laboratory for the measurements of alanine aminotransferase (ALT) and aspartate aminotransferase (AST) on the Dimension Vista ${ }^{\circledR}$ System (Siemens Healthcare Diagnostics Inc., Newark, Delaware, USA).

\section{Statistical analyses}


Statistical procedures were performed using SPSS (Version 22; SPSS, Inc., Chicago, IL) and statistical significance was set at $P \leq 0.05$. Independent samples $t$-tests or non-parametric test (the Mann Whitney U test) were used to compare physical and metabolic characteristics between obese black versus white adolescents. For variables that were not normally distributed, we used Pearson and partial correlation coefficients were employed to determine the associations of BMI, WC and abdominal AT (VAT and ASAT) to liver fat before and after controlling for age, gender, Tanner stage and total adiposity (\%). Forward stepwise multiple regression analyses were performed to quantify the independent contribution of WC to liver fat content. We used the probability of $\mathrm{F}<0.05$ as the criteria for variables to enter the model. In the correlation and stepwise multiple regression analyses, liver fat in both blacks and whites was log transformed to normalize their distribution. The receiver operating characteristic (ROC) curve analysis was used to determine the optimal cut-off value of WC to identify youth with NAFLD (liver fat $\geq 5.0 \%$ ). 


\section{RESULTS}

Participant characteristics are shown in Table 1. Despite similar age, gender and Tanner stage distribution, BMI, WC and total fat $(\mathrm{kg}, \%)$ between overweight and obese black and white adolescents, VAT, VAT/TAT (total abdominal adipose tissue) ratio, liver fat, and ALT were lower $(P<0.05)$ in blacks compared with their white peers.

\section{Liver fat content (\%) measured by ${ }^{1} H-M R S$}

As shown in Figure 1, the proportion of fatty liver (defined as liver fat $\geq 5 \%$ by ${ }^{1} \mathrm{H}-\mathrm{MRS}$ ) was lower $(P<0.01)$ in black $(n=5$ of $94,5.3 \%)$ versus white adolescents $(n=14$ of $58,24.1 \%)$. Fatty liver was present in $16.1 \%$ of black boys ( $n=5$ of 31 ) in contrast to $30.8 \%$ of white boys ( $n=8$ of 26 ); none in black girls $(n=0$ of 63 ) in contrast to $18.8 \%$ in white girls ( $n=6$ of 32 ).

\section{Correlations of BMI, WC and abdominal fat to liver fat}

In both black and white adolescents, BMI, WC, VAT and Abdominal SAT were associated $(P \leq 0.05)$ with liver fat (Table 2). After accounting for age, gender, Tanner stage and total adiposity (\%), only WC and VAT remained significant correlates of liver fat, by a similar magnitude, independent of race.

\section{Predictors of liver fat}

Stepwise multiple regression analyses were performed including liver fat as the dependent variable and age, gender, Tanner stage, BMI and $\mathrm{WC}$ as the independent variables (Table 3). In black adolescents, WC was the strongest predictor $(P<0.01)$ of liver fat, explaining $17.1 \%$ of the variance and followed by gender (male), explaining additional $5.6 \%$ of the 
variance. In white adolescents, WC was the only significant $(P<0.01)$ predictor of liver fat, explaining $40.8 \%$ of the variance.

ROC analyses revealed that in white obese adolescents, the optimal WC cutoff for detecting the presence of NAFLD was $101.5 \mathrm{~cm}$ with a sensitivity of $93 \%$ and a specificity of $80 \%$ [area under the curve (AUC): $0.847 \pm 0.052, P<0.001$ ]. Conversely, in black obese adolescents, the AUC was not significantly different from $0.5(0.749 \pm 0.080, P=0.061)$. 


\section{DISCUSSION}

We examined the associations between WC and liver fat in overweight and obese adolescents. Our findings suggest that in both black and white adolescents, WC is associated with liver fat in a similar magnitude to the associations between VAT and liver fat. Further, we observed WC is a significant predictor of liver fat, explaining $17 \%$ and $41 \%$ of the variance in black and white adolescents, respectively. These findings suggest that enlarged WC is a marker of increased liver fat in overweight/obese white and black adolescents.

A strong body of evidence suggests that enlarged WC is a predictor of cardiovascular diseases and insulin resistance in children and adolescents (Janssen et al. 2005; Lee et al. 2006a; Lee et al. 2006b; Burns and Arslanian 2009). According to the Bogalusa Heart Study, WC is an independent predictor of low HDL cholesterol, and high triglyceride, glucose and insulin levels and the metabolic syndrome in 2,597 black and white youth (5-18 yrs) (Janssen et al. 2005). In that study (Janssen et al. 2005), overweight youth (BMI 25-29.9 kg/m²) with larger WC were two times more likely to have high levels of triglycerides and insulin and the metabolic syndrome compared with overweight youth with smaller WC. Similarly, we previously demonstrated that WC contributes to the prediction of visceral adiposity and insulin sensitivity measured by hyperinsulinemic-euglycemic clamp, and the individual components of the metabolic syndrome independent of BMI percentiles in black and white youth (8-17 yrs) (Lee et al. 2006a; Lee et al. 2006b). In line with these findings, the current study demonstrated that enlarged WC is a significant risk factor for increased liver fat, particularly in obese white adolescents. Despite similar WC (blacks: $97.7 \mathrm{~cm}$ versus whites: $98.8 \mathrm{~cm}$ ), the two-fold higher liver fat in whites $(3.5 \%)$ than in blacks $(1.6 \%)$ may explain the greater ability of WC in predicting liver fat in obese white adolescents. 
Currently, the mechanisms by which WC explains increased liver fat are unclear in youth. However, we reported a strong association between WC and VAT in both black $(r=0.71)$ and white $(r=0.86)$ obese adolescents $(14)$, a finding similar to observations in Hispanic children $(r=0.65)$ (Ball et al. 2006) and pre-pubertal black and white children $(r=0.84)$ (Goran et al. 1998). The comparable relationships of WC (black: $r=0.43$, white: $r=0.64$ ) and VAT (black: $r=0.44$, white: $r=0.51$ ) with liver fat content observed in the current study may suggest that the ability of WC to predict liver fat could be explained, in part, by its ability to act as a surrogate marker of increased visceral adiposity, which is a well-known risk factor for NAFLD (Bjorntorp 1990; Despres and Lemieux et al. 2006).

In this study, we found that black adolescents (5.3\%) have significantly lower proportion of fatty liver compared with their white peers (24.1\%). These findings are consistent with previous studies reporting higher prevalence of NAFLD in white than in black youth based on liver histology (Schwimmer et al. 2006) and elevated serum ALT (Schwimmer et al. 2005). Further, our finding that obese white adolescents had a twofold higher liver fat as compared to obese black adolescents (3.6\% versus $1.8 \%)$ is consistent with a previous study by Liska et al. (Liska et al. 2007) who demonstrated virtually undetectable hepatic fat fraction (\%) in black obese adolescents $(n=17,0 \%)$ compared with their white peers $(n=21,17.5 \%)$ by employing MRI. Given a more favorable body fat distribution, it is unclear why black youth have more diabetogenic metabolic profiles than their white peers. Possibly, the pathogenesis of insulin resistance and the risk for T2DM in black youth may be linked with mechanisms unrelated to VAT and liver fat content (Liska et al. 2007).

Male gender has been associated with increased risk of NAFLD in children and adolescents (Schwimmer et al. 2005; Welsh et al. 2013). Schwimmer and colleagues 
(Schwimmer et al. 2005) demonstrated that unexplained ALT elevation is more common in boys (44\%) than in girls (7\%). Similarly, the most recent NHANES data (2007-2010) (Welsh et al. 2013) reported that the prevalence of suspected NAFLD is more than two times higher in boys (14.7\%) than in girls (6.3\%) among US adolescents (12-19 yrs). Although both studies (Schwimmer et al. 2005; Welsh et al. 2013) did not examine the influence of gender within each racial group, Schwimmer et al. (Schwimmer et al. 2005) speculated that differences in VAT and sex hormones may play a role in gender-differences in NAFLD. In the present study, fatty liver was present in $16 \%$ of black boys and not in black girls, in contrast to $31 \%$ of white boys and $19 \%$ of white girls. The lower amount of VAT in black girls $\left(53.8 \pm 19.5 \mathrm{~cm}^{2}\right)$ compared to black boys $\left(69.5 \pm 27.9 \mathrm{~cm}^{2}\right)$ and their white peers (boys: $90.3 \pm 36.1 \mathrm{~cm}^{2}$ and girls: $73.7 \pm 20.8$ $\mathrm{cm}^{2}$ ) could explain the lower liver fat content in black girls.

Although we observed that WC is associated with increased liver fat in both black and white adolescents, the strength of this association was more pronounced in white adolescents compared with their black peers. Indeed, WC was the single best predictor of liver fat in white adolescents, explaining $41 \%$ of the variance, whereas WC only explained $17 \%$ of the variance in black adolescents. In the present study, within whites, WC was significantly higher in adolescents with fatty liver compared with those without fatty liver $(108.2 \pm 6.4 \mathrm{~cm}$ versus 95.9 $\pm 10.2 \mathrm{~cm}, P<0.001)$, whereas no significant differences in $\mathrm{WC}(106.0 \pm 7.2 \mathrm{~cm}$ versus $97.2 \pm$ $11.0 \mathrm{~cm}, P=0.08)$ were observed between black adolescents with and without fatty liver. These findings have clinical implications and suggest that obese white adolescents with enlarged WC should be screened for NAFLD in clinical settings.

The strengths and limitations of this study warrant mention. We employed the gold standard non-invasive ${ }^{1} \mathrm{H}-\mathrm{MRS}$ technique to quantify liver fat and MRI to assess VAT. 
Although this is the first study to examine the ability of WC to predict liver fat employing ${ }^{1} \mathrm{H}-$ MRS in overweight and obese adolescents, our observations are based on a homogenous group of black and white adolescents with obesity. Whether our findings would remain true in other racial groups (such as Asian and Hispanic youth), pre-pubertal children, and normal-weight and severely obese youth are unknown. Given the cross-sectional design, small sample size and the differing sample size of the ethnic groups, studies with a larger sample size are needed to develop race-specific WC cutoffs to predict fatty liver in children and adolescents.

In conclusion, WC is associated with liver fat content in overweight/obese white and black adolescents. In particular, obese white adolescents with increased WC ( $\geq 102 \mathrm{~cm})$ should be screened for NAFLD in clinical settings. 


\section{ACKNOWLEDGEMENTS}

We would like to thank all participants and their parents who participated in this study and the nursing staff of the Pediatric Clinical and Translational Research Center at Children's Hospital of Pittsburgh of UPMC for their outstanding care of the participants. 
Funding source: This research was funded by the National Institutes of Health (Grant Numbers 5R01HL114857, 1R21DK083654-01A1, UL1 RR024153 and UL1TR000005), American Diabetes Association (7-08-JF-27), and Children's Hospital of Pittsburgh of UPMC (CochraneWeber Foundation and Renziehausen Fund) to Lee; Department of Defense (FA7014-02-2-001) and Richard L. Day endowed chair to Arslanian.

Financial Disclosure: The authors have no financial relationships relevant to this article.

Conflict of Interest Statement: The authors have no conflicts of interest relevant to this article. 


\section{REFERENCES}

Ball, G.D., Huang, T.T., Cruz, M.L., Shaibi, G.Q., Weigensberg, M.J., and Goran, M.I. 2006.

Predicting abdominal adipose tissue in overweight Latino youth. Int. J. Pediatr. Obes. 1(4):210-6.

Bjorntorp, P. 1990. "Portal" adipose tissue as a generator of risk factors for cardiovascular disease and diabetes. Arteriosclerosis, 10(4):493-6.

Brown, R.E., Kuk, J.L., Libman, I., Rivera-Vega, M., and Lee, S. 2015. Associations between visceral fat and liver fat with insulin sensitivity and metabolic risk in obese adolescents. Biochem. Cell. Biol. 93(5):466-71.

Burns, S.F. and Arslanian, S.A. 2009. Waist circumference, atherogenic lipoproteins, and vascular smooth muscle biomarkers in children. J. Clin. Endocrinol. Metab. 94(12):4914-22.

Despres, J.-P. and Lemieux, I. 2006. Abdominal obesity and metabolic syndrome. Nature, $14 ; 444(7121): 881-7$

Goran, M.I., Gower, B.A., Treuth, M., and Nagy, T.R. 1998. Prediction of intra-abdominal and subcutaneous abdominal adipose tissue in healthy pre-pubertal children. Int. J. Obes. Relat. Metab. Disord. 22(6):549-58. 
Janssen, I., Katzmarzyk, P.T., Srinivasan, S.R., Chen, W., Malina, R.M., Bouchard, C., et al. 2005. Combined influence of body mass index and waist circumference on coronary artery disease risk factors among children and adolescents. Pediatrics, 115(6):1623-30.

Kim, J.S., Le, K.A., Mahurkar, S., Davis, J.N., and Goran, M.I. 2012. Influence of elevated liver fat on circulating adipocytokines and insulin resistance in obese Hispanic adolescents. Pediatr. Obes. 7(2):158-64.

Kuk, J.L., Katzmarzyk, P.T., Nichaman, M.Z., Church, T.S., Blair, S.N., and Ross, R. 2006. Visceral Fat Is an Independent Predictor of All-cause Mortality in Men. Obesity (Silver Spring), $14(2): 336-41$.

Lavine, J.E. and Schwimmer, J.B. 2004. Nonalcoholic fatty liver disease in the pediatric population. Clin. Liver. Dis. 8(3):549-58, viii-ix.

Lee, S., Bacha, F., and Arslanian, S.A. 2006a. Waist circumference, blood pressure, and lipid components of the metabolic syndrome. J. Pediatr. 149(6):809-16.

Lee, S., Bacha, F., Gungor, N., and Arslanian, S.A. 2006b. Waist circumference is an independent predictor of insulin resistance in black and white youths. J. Pediatr. 148(2):188-94.

Lee, S., Bacha, F., Hannon, T., Kuk, J.L., Boesch, C., and Arslanian, S. 2012. Effects of aerobic versus resistance exercise without caloric restriction on abdominal fat, intrahepatic lipid, and 
insulin sensitivity in obese adolescent boys: a randomized, controlled trial. Diabetes, 61(11):2787-95.

Lee, S., Deldin, A.R., White, D., Kim, Y., Libman, I., Rivera-Vega, M., et al. 2013. Aerobic exercise but not resistance exercise reduces intrahepatic lipid content and visceral fat and improves insulin sensitivity in obese adolescent girls: a randomized controlled trial. Am. J. Physiol. Endocrinol. Metab. 15;305(10):E1222-9.

Lee, S., Guerra, N., and Arslanian, S. 2010. Skeletal muscle lipid content and insulin sensitivity in black versus white obese adolescents: is there a race differential? J. Clin. Endocrinol. Metab. 95(5):2426-32.

Limanond, P., Raman, S.S., Lassman, C., Sayre, J., Ghobrial, R.M., Busuttil, R.W., et al. 2004. Macrovesicular Hepatic Steatosis in Living Related Liver Donors: Correlation between CT and Histologic Findings. Radiology, 230(1):276-80.

Liska, D., Dufour, S., Zern, T.L., Taksali, S., Cali, A.M., Dziura, J., et al. 2007. Interethnic differences in muscle, liver and abdominal fat partitioning in obese adolescents. PLoS One. 27;2(6):e569.

Longo, R., Pollesello, P., Ricci, C., Masutti, F., Kvam, B.J., Bercich, L., et al. 1995. Proton MR spectroscopy in quantitative in vivo determination of fat content in human liver steatosis. J. Magn. Reson. Imaging. 5(3):281-5. 
Loomba, R., Sirlin, C.B., Schwimmer, J.B., and Lavine, J.E. 2009. Advances in pediatric nonalcoholic fatty liver disease. Hepatology, 50(4):1282-93.

Mishra, P. and Younossi, Z.M. 2007. Abdominal ultrasound for diagnosis of nonalcoholic fatty liver disease (NAFLD). Am. J. Gastroenterol. 102(12):2716-7.

Perseghin, G., Bonfanti, R., Magni, S., Lattuada, G., De Cobelli, F., Canu, T., et al. 2006. Insulin resistance and whole body energy homeostasis in obese adolescents with fatty liver disease. Am. J. Physiol. Endocrinol. Metab. 291(4):E697-703.

Schwimmer, J.B., Deutsch, R., Kahen, T., Lavine, J.E., Stanley, C., and Behling, C. 2006. Prevalence of fatty liver in children and adolescents. Pediatrics, 118(4):1388-93.

Schwimmer, J.B., McGreal, N., Deutsch, R., Finegold, M.J., and Lavine, J.E. 2005. Influence of gender, race, and ethnicity on suspected fatty liver in obese adolescents. Pediatrics, 115(5):e5615.

Schwimmer, J.B., Pardee, P.E., Lavine, J.E., Blumkin, A.K., and Cook, S. 2008. Cardiovascular risk factors and the metabolic syndrome in pediatric nonalcoholic fatty liver disease. Circulation, $15 ; 118(3): 277-83$. 
Szczepaniak, L.S., Nurenberg, P., Leonard, D., Browning, J.D., Reingold, J.S., Grundy, S., et al. 2005. Magnetic resonance spectroscopy to measure hepatic triglyceride content: prevalence of hepatic steatosis in the general population. Am. J. Physiol. Endocrinol. Metab. 288(2):E462-8.

Tanner, J.M. 1981. Growth and maturation during adolescence. Nutr. Rev. 39(2):43-55.

Welsh, J.A., Karpen, S., and Vos, M.B. 2013. Increasing prevalence of nonalcoholic fatty liver disease among United States adolescents, 1988-1994 to 2007-2010. J. Pediatr. 162(3):496-500.

Wicklow, B.A., Wittmeier, K.D., MacIntosh, A.C., Sellers, E.A., Ryner, L., Serrai, H., et al. 2012. Metabolic consequences of hepatic steatosis in overweight and obese adolescents. Diabetes Care, 35(4):905-10. 
TABLE 1. Participant characteristics

\begin{tabular}{|c|c|c|c|}
\hline & Black & White & $\boldsymbol{P}$ \\
\hline$n$ (boys/girls) & $94(31 / 63)$ & $58(26 / 32)$ & 0.098 \\
\hline Age (years) & $14.7 \pm 1.8$ & $14.5 \pm 1.5$ & 0.599 \\
\hline \multicolumn{4}{|l|}{ Tanner stage * } \\
\hline II-III/IV-V (n) & $8 / 85$ & $8 / 50$ & 0.229 \\
\hline Height $(\mathrm{cm})$ & $166.1 \pm 8.3$ & $167.8 \pm 9.0$ & 0.231 \\
\hline Weight (kg) & $95.5 \pm 17.9$ & $94.3 \pm 16.9$ & 0.696 \\
\hline BMI $\left(\mathrm{kg} / \mathrm{m}^{2}\right)$ & $34.4 \pm 4.3$ & $33.4 \pm 4.6$ & 0.176 \\
\hline BMI (percentile) & $98.3 \pm 1.5$ & $98.1 \pm 2.1$ & 0.256 \\
\hline Waist circumference $(\mathrm{cm})$ & $97.7 \pm 11.0$ & $98.8 \pm 10.8$ & 0.535 \\
\hline Total fat $(\mathrm{kg})$ & $40.8 \pm 10.2$ & $40.5 \pm 9.4$ & 0.837 \\
\hline Percent body fat (\%) & $42.5 \pm 5.2$ & $43.1 \pm 5.2$ & 0.629 \\
\hline $\operatorname{VAT}\left(\mathrm{cm}^{2}\right) * *$ & $59.0 \pm 23.6$ & $81.3 \pm 29.7$ & $<0.001$ \\
\hline Abdominal SAT $\left(\mathrm{cm}^{2}\right) * *$ & $486.7 \pm 142.5$ & $460.4 \pm 135.4$ & 0.265 \\
\hline VAT/TAT $* *$ & $0.11 \pm 0.04$ & $0.15 \pm 0.04$ & $<0.001$ \\
\hline Liver fat (\%) & $1.6 \pm 1.7$ & $3.5 \pm 4.0$ & $<0.001$ \\
\hline $\operatorname{ALT}(\mathrm{IU} / \mathrm{L}) \S$ & $17.2 \pm 7.6$ & $22.0 \pm 11.0$ & $<0.001$ \\
\hline $\operatorname{AST}(\mathrm{IU} / \mathrm{L}) \S$ & $21.3 \pm 6.5$ & $22.3 \pm 6.1$ & 0.064 \\
\hline
\end{tabular}

Mean \pm SD. NS, $P>0.1$.

VAT, visceral adipose tissue; SAT, subcutaneous adipose tissue; TAT: total abdominal adipose tissue; *one black girl's Tanner stage was not documented;

** $n=57$ in white; $\S n=93$ in black and $n=56$ in white. 
TABLE 2. Relationships of BMI, waist circumference and abdominal fat to liver fat in overweight and obese black and white adolescents

\section{Liver fat (\%)}

\begin{tabular}{lcccc}
\hline & \multicolumn{2}{c}{ Black } & \multicolumn{2}{c}{ White } \\
& Unadjusted & Adjusted $\S$ & Unadjusted & Adjusted $\S$ \\
\hline BMI $\left(\mathrm{kg} / \mathrm{m}^{2}\right)$ & $0.32^{*}$ & 0.18 & $0.48^{*}$ & 0.25 \\
Waist circumference $(\mathrm{cm})$ & $0.43^{*}$ & $0.28^{*}$ & $0.64^{*}$ & $0.47^{*}$ \\
VAT $\left(\mathrm{cm}^{2}\right)$ & $0.44^{*}$ & $0.31^{*}$ & $0.51^{*}$ & $0.35^{*}$ \\
Abdominal SAT $\left(\mathrm{cm}^{2}\right)$ & $0.20^{*}$ & 0.06 & $0.46^{*}$ & 0.20 \\
\hline
\end{tabular}

Liver fat in both blacks and whites were log transformed to normalize their distribution.

$\S$ Adjusted for age, gender, Tanner stage and total adiposity $(\%)$.

* Correlations are significant, $P \leq 0.05$. 
TABLE 3. Stepwise multiple regression analyses examining the independent associations between waist circumference and liver fat in overweight and obese black and white adolescents

\begin{tabular}{llclccc}
\hline Race & Dependent & Step & Independent & $\mathbf{R}^{2}$ & Sig. & Model \\
& variable & & Variable selected & Change & F Change & $\mathbf{R}^{2}$ \\
\hline Black & Liver fat & 1 & Waist circumference & 0.171 & $<0.001$ & \\
& & 2 & Gender (male) & 0.056 & 0.012 & $\mathbf{0 . 2 2 7}$ \\
& & & & & & \\
White & Liver fat & 1 & Waist circumference & 0.408 & $<0.001$ & $\mathbf{0 . 4 0 8}$ \\
\hline
\end{tabular}

Liver fat in blacks and whites was log transformed.

Independent variables entered in the stepwise regression model: Age, gender, Tanner stage, BMI and waist circumference. 


\section{FIGURE LEGENDS}

Figure 1. Proportion of fatty liver (defined as liver fat $\geq 5.0 \%$ by ${ }^{1} \mathrm{H}-\mathrm{MRS}$ ) in overweight and obese black and white adolescents. ${ }^{*} P<0.01$. 


\section{Page 25 of 25 \\ Figure 1}

Applied Physiology, Nutrition, and Metabolism

\section{Black $\square$ White}

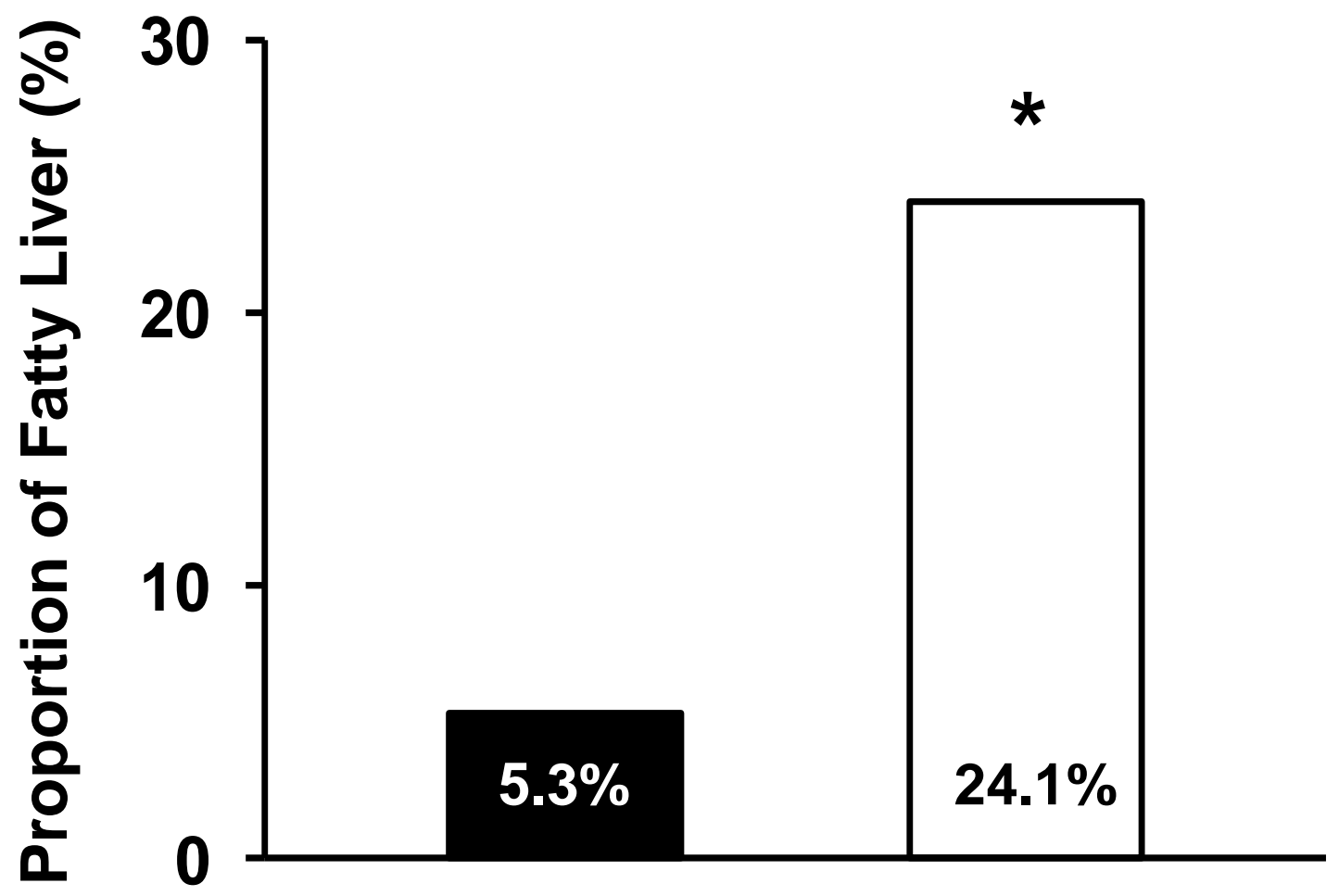

